\title{
The epidemiology of medium chain acyl-CoA dehydrogenase deficiency: An update
}

\author{
Scott D. Grosse, PhD ${ }^{1}$, Muin J. Khoury, MD, PhD ${ }^{2}$, Carol L. Greene, $M D^{3}$, Krista S. Crider, $P h D^{1}$,
} and Rodney J. Pollitt, $P h D^{4}$

\begin{abstract}
The most common fatty acid oxidation disorder, medium chain acyl-CoA dehydrogenase deficiency (MCADD), has become the focal point for the adoption of tandem mass spectrometry to detect it and related inborn errors of metabolism. This article updates a human genome epidemiology review of MCADD published in 1999. The focus of this update is on epidemiologic parameters rather than mutations associated with MCADD. Currently available information from screening studies on the frequency of detection of MCADD in newborns, as well as the frequency of homozygotes for the common mutation in the ACADM gene, is summarized. In the United States, the average incidence of the disorder is from 1 in 15,000 to 1 in 20,000 births, with individual states reporting frequencies from 1 in 10,000 to 1 in 30,000 births. In addition, a systematic review was undertaken of the published literature on the frequency of mortality and developmental disabilities among children with MCADD, both in screened and unscreened cohorts. It seems that in the absence of newborn screening for MCADD, premature death or serious disability occurs in $20 \%$ to $25 \%$ of children with the disorder. Systematic collection and analysis of follow-up data are still needed to ascertain the frequencies of outcomes in screened cohorts. Genet Med 2006:8(4):205-212.
\end{abstract}

Key Words: newborn screening, natural history, MCAD deficiency, epidemiology, metabolic disease

The recent incorporation of medium chain acyl-CoA dehydrogenase deficiency (MCADD) in newborn screening programs has facilitated the expansion of epidemiologic knowledge of this treatable disorder. This review of the epidemiology of MCADD incorporates population-based data derived from the use of tandem mass spectrometry (MS/MS) by newborn screening programs. In 1999, when a previous human genome epidemiology (HuGE) review of MCADD was published, ${ }^{1}$ screening for the disorder in the United States was restricted to three states, and almost all information came from clinical case series or pilot studies. As of June 2005, screening for MCADD had been implemented in 32 states, and implementation planned in 5 additional states. ${ }^{2}$ These states collectively represent approximately $78 \%$ of all births in the United States.

Despite the increase in screening, understanding of the natural history of unscreened MCADD remains limited. ${ }^{1,3,4}$ Screening for a treatable disorder by definition cannot provide information on the course of the disorder in the absence of screening. The major preventable outcomes of concern are

\footnotetext{
From the ${ }^{1}$ National Center on Birth Defects and Developmental Disabilities, Coordinating Center for Health Promotion, Centers for Disease Control and Prevention, Atlanta, Ga; ${ }^{2}$ Office of Genomics and Disease Prevention, Coordinating Center for Health Promotion, Centers for Disease Control and Prevention, Atlanta, Ga; ${ }^{3}$ University of Maryland School of Medicine, Baltimore, Md; ${ }^{4}$ Children's Hospital, Sheffield, United Kingdom.

Scott Grosse, 1600 Clifton Road, Mail Stop E-87, Atlanta, GA 30333.

Submitted for publication October 19, 2005.

Accepted for publication January 2, 2006.

The findings and conclusions are those of the authors and do not represent the views of the Centers for Disease Control and Prevention.
}

DOI: 10.1097/01.gim.0000204472.25153.8d death and intellectual disability. Death is reported to occur in up to $25 \%$ of symptomatic individuals with MCADD., ${ }^{1,5}$ The frequency of death in untreated MCADD is said to be as high as $50 \%{ }^{6,7}$ or as low as $2 \% .^{8}$ This variation highlights the need for more precise numerical estimates for quantitative policy analysis.

\section{METHODS}

We conducted a systematic search for relevant studies published since the appearance of the previous HuGE review on MCADD. ${ }^{1}$ We conducted a systematic search of Medline for articles on MCADD published since 1999 in the English language, using as search terms either "medium chain acyl-CoA dehydrogenase deficiency" or "tandem mass spectrometry" together with "newborn screening." In deciding which articles to review, we excluded diagnostic studies, studies of gene mutations in small population samples, and case reports. Also, we scanned newborn screening conference abstracts and proceedings and the reports of state newborn screening programs available on the World Wide Web for additional data on the reported frequency of MCADD cases detected by newborn screening programs in the United States.

\section{Gene and gene variants}

MCADD is a mitochondrial fatty acid oxidation disorder that results from inactivity or deficiency of the medium chain acyl-CoA dehydrogenase (MCAD) protein. The MCAD protein is an enzyme that catalyzes the beta-oxidation of fatty ac- 
ids. This process is critical to the formation of ketone bodies in the liver, which provide an alternative energy source during periods of prolonged fasting or increased energy demands.

The MCAD protein is coded by the ACADM (alias MCAD) gene (OMIM number 607008), which is located at chromosome $1 \mathrm{p} 31$. More than 30 mutations in this gene have been identified, most of which are missense mutations in which one amino acid is substituted for another at a specific codon. ${ }^{9}$ The most common mutation involves an adenine-to-guanine $(\mathrm{A} \rightarrow \mathrm{G})$ transition at coding position 985 on the gene (OMIM variant number 607008.0001 commonly referred to as $985 \mathrm{~A} \rightarrow \mathrm{G}$ or A985G) resulting in a substitution of glutamate for lysine at position 329 of the precursor protein (K329E or Lys329Glu) that corresponds to position 304 in the mature protein (K304E). Two copies of this common mutation are typically found in $80 \%$ of European-origin individuals diagnosed with MCADD on the basis of clinical symptoms. ${ }^{9,10}$ Other mutations predominate in non-European populations, and additional mutations may be identified as screening becomes increasingly widespread. ${ }^{11,12}$

The pattern of genotypes differs between screened and clinically detected cohorts, with a lower frequency of A985G homozygosity among individuals with MCADD detected by screening (Table 1). Different studies reporting on children identified prospectively through screening report that from $36 \%$ to $71 \%$ (mean frequency of $55 \%$ ) are homozygous for the A985G mutation, with a $95 \%$ confidence interval from $47 \%$ to $63 \%$ (Table 1). We report exact binomial confidence intervals, which are appropriate for estimates of proportions or percentages, for parameters in Tables 1, 2, 4, and 5 to give an indication of the degree of uncertainty surrounding each estimate.

Results from screened populations show the presence of a common missense mutation (T199C) that has not been found

\section{Table 1}

Frequency of homozygotes for A985G mutation among MCADD cases detected through screening newborn dried blood spots using tandem mass spectrophotometry

\begin{tabular}{|c|c|c|c|c|}
\hline Citation & $\begin{array}{l}\text { No. } \\
\text { cases }\end{array}$ & $\begin{array}{c}\text { No. } \\
\text { homozygotes }\end{array}$ & $\begin{array}{l}\text { Proportion of } \\
\text { homozygotes }\end{array}$ & $\begin{array}{l}95 \% \text { confidence } \\
\text { intervals }^{a}\end{array}$ \\
\hline $\begin{array}{l}\text { Andresen et al. } \\
2001^{13}\end{array}$ & $60^{b}$ & 39 & $65.0 \%$ & $51.5 \%-76.9 \%$ \\
\hline $\begin{array}{l}\text { Zytkovicz et al. } \\
2001^{43}\end{array}$ & 10 & 4 & $40.0 \%$ & $12.2 \%-73.8 \%$ \\
\hline $\begin{array}{l}\text { Carpenter et al. } \\
2001^{17}\end{array}$ & 11 & 4 & $36.4 \%$ & $10.9 \%-69.2 \%$ \\
\hline $\begin{array}{l}\text { Zschocke et al. } \\
2001^{18}\end{array}$ & 14 & 10 & $71.4 \%$ & $41.9 \%-91.6 \%$ \\
\hline $\begin{array}{l}\text { Maier et al. } \\
2005^{11}\end{array}$ & 57 & 27 & $47.4 \%$ & $34.0 \%-61.0 \%$ \\
\hline Pooled & 152 & 84 & $55.3 \%$ & $47.0 \%-63.3 \%$ \\
\hline
\end{tabular}

${ }^{a}$ Ninety-five percent exact binomial confidence intervals, calculated with STATA v. 8 (StataCorp, College Station, Tex).

${ }^{b}$ The original article reported 62 cases of MCADD based on acylcarnitinepositive newborn samples; however, not all were confirmed by diagnostic criteria. MCADD, medium chain acyl-CoA dehydrogenase deficiency. in patients with clinical disease. ${ }^{11,13}$ The T199C allele is relatively common $(1 / 500$ as determined by a random screen of 1,000 blood spots), and in vitro studies show reduced enzyme activity, although not necessarily of physiologic significance. ${ }^{13}$ Analysis of data from a large screened population found the T199C allele in combination with the A985G allele or with other rare mutations in $16 \%$ of the screen-positive individuals. ${ }^{11}$ The absolute disease risk for children who screen positive and carry the T199C allele is unknown at this time.

Children with MCADD detected through screening can be expected to have a lower average risk for developing symptomatic disease because they are less likely to have genotypes associated with clinical symptoms. It should be noted that the genotype-phenotype correlation is limited, and even among A985G homozygotes the spectrum of outcomes is variable. ${ }^{14}$ The association is likely dependent on a variety of genetic and environmental factors. For example, not all affected individuals experience sufficient metabolic stress in the course of childhood illnesses to precipitate a metabolic crisis.

\section{Case definition and diagnostic criteria}

Criteria for the diagnosis of MCADD have evolved over time. Demonstration of deficient enzymatic activity is the gold standard for diagnosis of this fatty acid oxidation disorder. The high specificity of a diagnostic acylcarnitine profile obtained using MS/MS had led to decreased use of enzyme assays for confirmation in the clinical setting. ${ }^{15}$ However, certain individuals develop secondary carnitine deficiency that suppresses the diagnostic acylcarnitine profile and can cause falsenegative diagnostic results. ${ }^{16}$ Before the advent of MS/MS, diagnosis of probands almost invariably followed clinical presentation (unexplained lethargy, altered consciousness, hypoglycemia, encephalopathy, or fatty liver on autopsy) supported by a combination of biochemical abnormalities.

Not all individuals with MCADD develop such a clinical presentation, whether a severe metabolic crisis or milder symptoms. This causes some confusion as to whether the MCADD phenotype consists of specific clinical signs and symptoms or the biochemical evidence of an enzyme disorder. For the purposes of this review, the authors followed established clinical practice in using the biochemical definition of the MCADD phenotype.

Use of variable diagnostic criteria for MCADD complicates comparison of data from multiple sources. Some screening programs require confirmatory biochemical tests. ${ }^{16,17}$ The presence of two severe mutations on the ACADM gene, that is, missense or stop mutations resulting in no residual enzymatic activity, is considered diagnostic, although not all individuals who are diagnosed with MCADD undergo molecular testing. Some consider repeat abnormal acylcarnitine screening profiles using the same blood spots to be diagnostic, although it is not clear that all children identified through such techniques would meet standard diagnostic criteria.

Some people who are compound heterozygotes for $M C A D$ mutations have a mild or benign phenotype. ${ }^{17}$ German inves- 
Table 2

Estimated incidence of MCAD from screening of newborn blood spots

\begin{tabular}{|c|c|c|c|c|c|c|}
\hline Citation & Country & State(s) & No. screened & No. cases & Incidence & $95 \%$ confidence intervals ${ }^{a}$ \\
\hline Pourfarzam et al. $2001^{33}$ & England & & 100,600 & 8 & $1: 12,600$ & $1: 6,400-1: 29,200$ \\
\hline Shigematsu et al. $2002^{21}$ & Japan & & 102,200 & 2 & $1: 51,100$ & $1: 14,100-1: 422,000$ \\
\hline Wilcken et al. $2003^{25}$ & Australia & $\begin{array}{l}\text { New South Wales and Australian } \\
\text { Capital Territory }\end{array}$ & 362,000 & 17 & $1: 21,300$ & $1: 13,300-1: 36,500$ \\
\hline Sander et al. $2001^{45}$ & Germany & $\begin{array}{l}\text { Lower Saxony, North } \\
\text { Rhine-Westphalia, etc. }\end{array}$ & 283,408 & 29 & $1: 9,800$ & $1: 6,800-1: 14,600$ \\
\hline Schulze et al. $2003^{19}$ & Germany & Baden-Wurttemberg & 250,000 & 16 & $1: 15,600$ & $1: 11,900-1: 40,300$ \\
\hline Maier et al. $2005^{11}$ & Germany & Bavaria & 524,287 & 62 & $1: 8,100$ & $1: 6,300-1: 10,400$ \\
\hline \multirow[t]{2}{*}{ Hoffman et al. $2004^{26}$} & Germany & Baden-Wurttemberg and Bavaria & 382,247 & 29 & $1: 13,100$ & $1: 10,300-1: 18,900$ \\
\hline & Germany $^{b}$ & Pooled & $1,057,695$ & 107 & $1: 9,900$ & $1: 8,200-1: 12,100$ \\
\hline Chace et al. $2002^{22}$ & US & $\mathrm{Pa}, \mathrm{NC}$, etc. & $1,019,602$ & 65 & $1: 15,700$ & $1: 12,300-1: 20,300$ \\
\hline Zytkovicz et al. $2001^{43}$ & US & Mass, $\mathrm{Me}$ & 184,000 & 10 & $1: 18,400$ & $1: 10,010-1: 38,314$ \\
\hline Insinga et al. $2002^{46}$ & US & Wis & 155,500 & 7 & $1: 22,200$ & $1: 10,800-1: 55,200$ \\
\hline Lorey $2004^{47}$ & US & Calif & 354,074 & 13 & $1: 27,200$ & $1: 15,900-1: 51,300$ \\
\hline Frazier $2004^{48}$ & US & NC & 119,201 & 12 & $1: 9,900$ & $1: 5,700-1: 19,200$ \\
\hline NY Annual Report $2003^{49}$ & US & NY & 250,153 & 12 & $1: 20,800$ & $1: 11,900-1: 40,300$ \\
\hline Indiana Annual Report $2002^{50}$ & US & Idaho & 84,618 & 2 & $1: 42,300$ & $1: 11,700-1: 350,000$ \\
\hline \multirow[t]{2}{*}{ North West Regional States ${ }^{48}$} & US & Ore, Ala, Hawaii, Nev & 147,811 & 15 & $1: 9,900$ & $1: 6,000-1: 17,600$ \\
\hline & US & Pooled & $1,713,176$ & 95 & $1: 17,000$ & $1: 14,400-1: 20,300$ \\
\hline
\end{tabular}

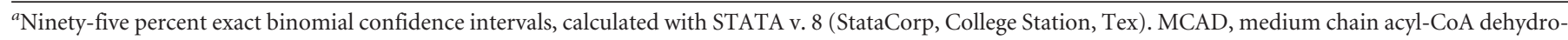
genase.

${ }^{b}$ Pooled estimates for Germany based on studies 11, 19, and 45 to avoid double counting the same observations.

tigators proposed an algorithm based on residual enzymatic activity in fibroblasts, lymphocytes, or both; mutation analysis; and phenylpropionate loading. ${ }^{18,19}$ According to that algorithm, classic MCADD is indicated by less than $5 \%$ residual enzymatic activity, the presence of two severe mutations, or a pathologic phenylpropionate loading result. Mild MCADD is associated with $5 \%$ to $35 \%$ residual enzymatic activity, mild mutations, and normal phenylpropionate loading. German data have indicated that one-fourth of infants with MCADD detected by newborn screening have mild phenotypes and that the other three-quarters have classic MCADD. ${ }^{19}$

\section{Incidence and prevalence}

Accurate assessment of the incidence of MCADD requires analysis of test results from a birth cohort representative of a geographic population. Data on the frequency of MCADD cases detected through newborn screening are summarized in Table 2. The estimated frequency of MCADD at birth ranges from 1 in 10,000 to 1 in 27,000 among populations of mostly European descent and is less common in populations of nonEuropean origin. No cases of MCADD were found among 79,171 newborns screened in Korea, ${ }^{20}$ and two cases of MCADD were found among 102,200 infants screened in Japan. $^{21}$

A U.S. screening study, comprising data largely from the states of Pennsylvania and North Carolina, reported an average incidence of 1 in 15,700 births. ${ }^{22}$ On the basis of U.S. studies reporting screening program data, the pooled estimate of the incidence of MCADD for the United States is 1 in 17,000 births, with an approximate $95 \%$ confidence interval from 1 in 15,000 to 1 in 20,000. Substantial variation is reported among states, with the incidence in individual states varying from approximately 1 in 10,000 to approximately 1 in 30,000. States cannot reliably predict the number of MCADD cases on the basis of national incidence data or screening data from another state. None of the published screening studies from the United States reported cases by race or ethnicity. However, the incidence or birth prevalence for an individual state is likely to vary with racial and ethnic composition.

Because of variable and nonspecific clinical presentation, MCADD often is not recognized and, consequently, is underdiagnosed at the population level in the absence of screening. Three surveillance studies surveyed metabolic centers, pediatric practices, and diagnostic laboratories to determine the prevalence of diagnosed metabolic disorders in birth cohorts for defined populations. The prevalence of clinically diagnosed MCADD among cohorts of children in geographically defined populations was 1 in 30,900 in England ${ }^{23}$; 1 in 42,200 in German states not using MS/MS newborn screening24; and 1 in 47,300 in New South Wales, Australia, from 1990 to $1994 .^{25}$ The German study used a capture-recapture analysis based on two independent reporting sources to estimate that the true 
Table 3

Comparison of active surveillance and newborn screening prevalence estimates of MCADD

\begin{tabular}{lccc}
\hline Country & $\begin{array}{c}\text { Prevalence of } \\
\text { clinically detected } \\
\text { cases (A) }\end{array}$ & $\begin{array}{c}\text { Prevalence from } \\
\text { newborn screening } \\
(\mathrm{B})\end{array}$ & $\begin{array}{c}\text { Ratio } \\
\text { A/B }\end{array}$ \\
\hline England & $1: 30,700^{23}$ & $1: 12,700^{33}$ & 0.41 \\
Southern Germany & $1: 38,000^{26}$ & $1: 13,100^{26}$ & 0.35 \\
Australia & $1: 47,300^{25}$ & $1: 21,300^{25}$ & 0.45 \\
\hline
\end{tabular}

MCADD, medium chain acyl-CoA dehydrogenase deficiency.

prevalence of clinically reported cases was 1 in $38,000.24,26$ In The Netherlands, a retrospective cohort study found an average prevalence of MCADD of 1 in 27,400.27

With three countries (Australia, England, and Germany) it was possible to compare prevalence data from active surveillance with that from newborn screening. In each of those countries, the number of children with MCADD detected by screening was more than twice the number diagnosed on the basis of symptoms. With the exclusion of children identified with MCADD on the basis of family history, the ratio of clinically diagnosed prevalence in unscreened cohorts and screened cohorts ranged from 0.35 to 0.45 , respectively (Table 3 ).

In the United States, MCADD seems even less likely to be diagnosed in the absence of newborn screening. Researchers affiliated with a large health plan in California reported no diagnosed MCADD cases among their members. ${ }^{8}$ In a comparative study, MCADD cases accounted for $40 \%$ of all cases of metabolic disorders detected by MS/MS screening in Pennsylvania, Massachusetts, and Maine, but for only $15 \%$ of cases of the same diseases detected on the basis of clinical symptoms in three other New England states. ${ }^{28}$

\section{Natural history}

The baseline for calculating the potential benefits of newborn screening is the natural history of the disorder. The natural history includes the pattern of clinical signs, symptoms, and sequelae and outcomes such as mortality and disability among individuals in whom the disease is not detected before the emergence of clinical symptoms. It should be recognized that this is not fixed but depends on the state of clinical awareness and therapeutic practices.

\section{Clinical symptoms}

Because fatty acid oxidation is important for energy production during fasting or increased energy expenditure, fasting or stress in individuals with MCADD can lead to a variety of complications. These include hypoketotic hypoglycemia, hypotonia, lethargy, and vomiting. This can progress to seizures, encephalopathy, coma, and death with an accumulation of toxic metabolites. ${ }^{14,15}$ Because signs and symptoms are highly variable, no consistent definition of what is regarded as symptomatic MCADD is used in the literature.

Table 4

Percentage of children diagnosed symptomatically with MCAD who died

\begin{tabular}{|c|c|c|c|c|c|c|}
\hline Citation & Country & Time period & No. MCAD probands & No. deaths & $\%$ deaths & $95 \%$ confidence intervals ${ }^{a}$ \\
\hline Touma and Charpentier ${ }^{51}$ & France & & 65 & 17 & $26.2 \%$ & $16.0 \%-38.5 \%$ \\
\hline Iafolla et al. ${ }^{38}$ & US, others & & 120 & 23 & $19.2 \%$ & $12.6 \%-27.4 \%$ \\
\hline Wilcken et al. ${ }^{30}$ & Australia & 1975-1993 & 16 & 2 & $12.5 \%$ & $1.6 \%-38.3 \%$ \\
\hline Pollitt and Leonard ${ }^{23}$ & UK & 1994-1996 & 62 & 10 & $16.1 \%$ & $8.0 \%-27.7 \%$ \\
\hline Wilson et al. ${ }^{37 b}$ & UK & 1993-1997 & 12 & 1 & $8.3 \%$ & $0.2 \%-38.5 \%$ \\
\hline Klose et al. ${ }^{24}$ & Germany & 1999-2000 & 20 & 2 & $10.0 \%$ & $1.2 \%-31.7 \%$ \\
\hline
\end{tabular}

${ }^{a}$ Ninety-five percent exact binomial confidence intervals, calculated with STATA v. 8 (StataCorp, College Station, Tex).

${ }^{b}$ Data in Wilson et al. ${ }^{37}$ were included in report by Pollitt and Leonard. ${ }^{23}$ MCAD, medium chain acyl-CoA dehydrogenase.

Table 5

Percentage of children surviving acute metabolic crises with MCAD who were assessed with serious neurocognitive impairments

\begin{tabular}{|c|c|c|c|c|c|}
\hline Citation & Country & No. MCAD survivors & No. severe disability & $\%$ severe disability & $95 \%$ confidence intervals $^{a}$ \\
\hline Iafolla et al. ${ }^{38}$ & US, others & 73 & 12 & $16.4 \%$ & $8.8 \%-27.0 \%$ \\
\hline Wilcken et al. ${ }^{30}$ & Australia & 15 & 1 & $6.7 \%$ & $1.7 \%-31.9 \%$ \\
\hline Pollitt and Leonard ${ }^{23}$ & UK & 36 & 3 & $8.3 \%$ & $1.8 \%-22.5 \%$ \\
\hline Wilson et al. ${ }^{37}$ & UK & 30 & 2 & $6.7 \%$ & $1.7 \%-31.9 \%$ \\
\hline Waisbren et al. ${ }^{28}$ & US & 5 & 0 & $0 \%$ & \\
\hline Pooled estimate ${ }^{b ; 29,14,27}$ & & 56 & 4 & $7.1 \%$ & $2.0 \%-17.3 \%$ \\
\hline
\end{tabular}

${ }^{a}$ Ninety-five percent exact binomial confidence intervals, calculated with STATA v. 8 StataCorp (College Station, Tex).

${ }^{b}$ Data in Wilson et al. ${ }^{37}$ were included in report by Pollitt and Leonard ${ }^{23}$ and are excluded from pooled estimate. MCAD, medium chain acyl-CoA dehydrogenase. 
The age of presentation is variable. The majority of symptomatic cases present from 3 months to 3 years of age. ${ }^{1}$ However, MCADD can present in the newborn period, and clinicians in neonatal units must be alert to early presentation to prevent death or neurologic damage. ${ }^{29}$ In New South Wales, 8 of 20 children with MCADD presented with acute hypoglycemia during the first 3 days of life, before the time when newborn screening results could be made available. ${ }^{30}$

The probability of an individual with MCADD detected by screening developing a metabolic decompensation crisis in the absence of screening is as high as $0.75 .^{31,32}$ One retrospective study, on the basis of MS/MS analysis of 100,600 stored blood spot specimens, identified eight children with MCADD at ages 7 to 9 years..$^{33}$ Of the eight cases, one had died, and two others had been clinically diagnosed with MCADD, both having experienced episodes of encephalopathy. Of the remaining five children, one child had had episodes of encephalopathy and two others had had episodes of hypoglycemia in infancy. The other two children remained asymptomatic. Thus, four of eight (50\%) had experienced serious metabolic crises resulting in encephalopathy or death, and six of eight $(75 \%)$ had experienced clinical symptoms.

The frequency with which children with biochemical MCADD develop serious, life-threatening symptoms can also be assessed by using information on the older siblings of probands detected as newborns through screening. For example, Waisbren and colleagues, through testing family members of 20 infants detected through screening, identified seven older surviving siblings with MCADD, of whom four had shown symptoms (hypoglycemia and extreme lethargy) of MCADD, whereas the other three remained asymptomatic. ${ }^{28}$ That excludes older siblings who might have died of MCADD. Pollitt and Leonard reported that four of six older siblings with MCADD experienced symptoms, and that an additional four siblings died with symptoms compatible with MCADD. ${ }^{23}$

\section{Mortality}

Death has been reported to occur in up to $25 \%$ of children with MCADD not previously diagnosed who experience episodes of acute metabolic decompensation. ${ }^{1,5}$ Published reports of the frequency of death among children who are index cases of MCADD are found in Table 4. Pourfarzam et al. found one death among four children with MCADD who presented with acute metabolic decompensation (25\%). ${ }^{33}$ Pollitt and Leonard reported that 10 of 46 children with MCADD who presented with acute symptoms died (21.7\%). ${ }^{23}$

The calculated mortality risk in MCADD depends on the length of follow-up, because deaths associated with the disorder can occur through at least 3 years of age. The effect of length of follow-up has not been adequately addressed in the published literature on mortality in MCADD. One study reported 2 infant deaths among 27 patients (9\%) with clinically detected MCADD at the Children's Hospital of Philadelphia. ${ }^{34}$ The risk of death through 3 years of age was $26 \%$ in the same cohort (Charles Venditti, MD, PhD, personal communication, 2003). The cumulative risk of death to 12 months of age was
13.0\% (6/46) in the English cohort of Pollitt and Leonard, compared with $21.7 \%$ at 3 years of age. ${ }^{23}$ A study from Germany reported that only $2(10 \%)$ of 20 children clinically diagnosed with MCADD had died, but the length of follow-up was short, perhaps less than 12 months. ${ }^{24}$

The overall mortality rate among all children with MCADD is necessarily lower than that among those who experience metabolic crises, because a fraction of individuals with the disorder do not manifest signs and symptoms in childhood. If the mortality rate among children who experience an acute metabolic crisis were $25 \%$ and the probability of such a crisis were $50 \%$ to $75 \%$, overall cohort mortality would be between $12 \%$ and 20\%. In the British cohort ascertained by Pollitt and Leonard, there were 10 deaths among 62 probands, for an overall risk of death of $16.1 \% .^{23}$ Similarly, in the small group of eight children with MCADD detected by Pourfarzam et al., ${ }^{33}$ there was one death (12.5\%).

The number of deaths attributed to a diagnosis of MCADD is probably understated in certain studies because infants or children who die of MCADD-related causes might have the cause of death incorrectly listed as sudden infant death syndrome, Reye syndrome, hepatitis, poisoning, or unknown causes unless metabolic testing is conducted on autopsy specimens. ${ }^{35}$ Among 7,058 postmortem blood samples obtained from children in the United States and Canada with unexpected deaths, 23 had evidence of MCADD deficiency. ${ }^{35} \mathrm{~A}$ follow-up study that analyzed postmortem blood spots from 793 children in Virginia who died before 3 years of age and whose deaths were referred to a medical examiner during 1996 to 2001 revealed that two had evidence of MCADD by acylcarnitine analysis, both of whose diagnoses were subsequently confirmed by genotyping. ${ }^{36}$

The data from the Virginia study can be used to establish a lower bound on the risk of undiagnosed mortality from MCADD in young children. Approximately 550,000 births took place in Virginia from 1996 to 2001. By assuming a prevalence of 1 in 16,000, one would expect 35 births in Virginia affected by MCADD during this period. Dividing 2 deaths by 35 expected cases, we project that undiagnosed deaths occurred in $6 \%$ of children born with MCADD during this period. This is likely to be an underestimate, because other MCAD-related deaths might not have been subject to postmortem examination because they were assigned another cause of death such as infectious disease.

The frequency of deaths among older siblings of children with MCADD seems elevated relative to that among probands. For example, Wilcken and colleagues reported that $3(75 \%)$ of 4 Australian siblings presumed to have had MCADD had died, compared with $2(12.5 \%)$ of 16 probands. ${ }^{30}$ Similarly, Wilson and colleagues reported that in England 9 (88\%) of 11 older siblings who likely had MCADD had deaths consistent with MCADD. ${ }^{37}$ One reason for the higher than expected numbers of deaths in older siblings is that the typical nonspecific symptoms of MCADD might be more likely to be diagnosed correctly in a child with a family history of sibling death. 


\section{Neurologic complications}

Children who experience acute metabolic decompensation are at elevated risk of neurologic impairment and varying degrees of disability. Up to one-third of survivors of metabolic crises in MCADD are reported to experience some form of developmental delay. Differences in the classification of neurologic impairments make comparisons across studies difficult. Iafolla et al. reported that among 73 patients older than 2 years of age, one or more abnormal assessments were found in $32 \%$, including speech and language delay (22\%), developmental disability (16\%), and behavioral problems $(15 \%){ }^{38}$ Wilcken et al. likewise reported one-third of MCADD survivors in Australia to have some developmental problems. ${ }^{30}$ Two studies from the United Kingdom both reported developmental problems in one-sixth of survivors of acute crises. ${ }^{23,37}$

Measures of intellectual disability or mental retardation, defined as IQ less than 70, seem more comparable (Table 5). By excluding the study by Iafolla and colleagues, ${ }^{38}$ the reported frequency of cognitive disability in children with MCADD detected symptomatically is $7 \%$ to $8 \%$. Pooling across studies, the probability of intellectual disability is $7.1 \%$, with a $95 \%$ confidence interval from $2.0 \%$ to $17.3 \%$. Waisbren et al. found no cases of marked developmental delay among five children with MCADD detected on the basis of clinical symptoms, which is consistent with a risk of $10 \%$ or less. ${ }^{28}$

Not all cases of intellectual disability in children with MCADD can be attributed to the metabolic defect. The background rate of mental retardation in children is approximately $1 \% .{ }^{39}$ If one subtracts the background rate, the estimate of the risk of mental retardation or intellectual disability among children with MCADD diagnosed on the basis of clinical symptoms is $6 \%$ in the absence of early, asymptomatic detection.

The other developmental disability that has been associated with MCADD is cerebral palsy. In a series of 97 surviving patients with MCADD reported by Iafolla et al., 9\% had been diagnosed with cerebral palsy. ${ }^{38}$ The investigators did not report how many children with cerebral palsy also had cognitive disability. In general, studies show that approximately twothirds of children with cerebral palsy also have a diagnosis of mental retardation. ${ }^{39}$ This suggests an additional 3\% risk of cerebral palsy without cognitive impairment, for a total of $9 \%$ of children with mental retardation, cerebral palsy, or both attributable to the effects of MCADD.

We combined the best estimate of the attributable risk of intellectual disability among surviving children with clinically diagnosed MCADD (6\%) and the risk of cerebral palsy without intellectual disability (3\%) to derive an overall estimate of developmental disability severe enough to require lifelong treatment (9\%). This estimate is imprecise, given the wide confidence interval for intellectual disability $(2.0 \%-17.0 \%)$ and the even less precise estimate of the frequency of cerebral palsy. The estimate applies to children who experience a metabolic crisis, which is from $50 \%$ to $75 \%$ of all children with MCADD in the absence of screening. Logically, this implies $5 \%$ to $7 \%$ of children with MCADD would develop some type of frank de- velopmental disability in the absence of screening, although this does not take into account the statistical uncertainty with regard to the underlying estimates. Further, this estimate does not take into account milder developmental delays or behavioral problems that can manifest among survivors of metabolic crises.

\section{Prevention of symptoms and complications}

The reduced ability to metabolize medium-chain fatty acids becomes problematic when individuals are faced with extra metabolic demands or reduced dietary inputs. Most often, stress induced by fasting or infection, during which the demands on fatty acid oxidation are particularly high, leads to symptomatic presentation. Lengthy fasting or the presence of infection or recent immunization typically precedes metabolic crises in MCADD. ${ }^{40}$

It is widely believed that adverse outcomes in MCADD can largely be prevented through avoidance of fasting, along with close attention by parents and clinicians during periods of infection and after immunizations. Energy-containing drinks should be consumed during periods of infection and anorexia. Hospitalization for administration of intravenous fluids is required if oral feeding attempts are unsuccessful. The only deaths after diagnosis of MCADD that have been reported in the published literature from the United States occurred in children in whom the diagnosis was made late or disease-management guidelines were not necessarily followed. ${ }^{13,40}$

Information on long-term outcomes in children with MCADD detected through newborn screening is still lacking. ${ }^{4,25,41}$ However, there are indications that the risk of mortality after newborn screening for MCADD is real. A recent report from Germany examined the outcomes of 29 patients detected by screening who were homozygous for the A985G mutation. ${ }^{42}$ Unfortunately, two patients died at 10 months of age, despite knowledge of the MCADDD diagnosis. ${ }^{42}$ Long-term follow-up investigations in New England have likewise revealed two deaths among children identified by newborn screening whose MCADD diagnoses were confirmed by metabolic specialists (number of children diagnosed is not reported); the details of the circumstances of these deaths are under investigation (personal communication, New England Newborn Screening Program).

Severe metabolic crises sometimes occur before the reporting of screening results, further reducing the number of preventable deaths resulting from newborn screening. Pollitt and Leonard reported one neonatal death in an infant whose older sibling had already been diagnosed with MCADD. ${ }^{23}$ Among 23 infant deaths with MCADD detected by postmortem MS/MS analysis, 3 occurred during the first 7 days of life. ${ }^{35}$ Presumably, these deaths could not have been averted by newborn screening. On the other hand, early neonatal deaths with MCADD might be preventable through increased clinical awareness of fatty acid oxidation disorders, which can occur as a result of their inclusion in newborn screening panels.

Studies of screened cohorts with MCADD have reported no neurologic impairments. ${ }^{13,25,28,43}$ However, none of these 
studies administered a validated test of general cognitive ability required to classify intellectual disability. One study assessed 20 children with MCADD identified through newborn screening from 5 to 29 months of age, with a median age of 9 months. ${ }^{28}$ None of the children with MCADD had mental index scores below the normal range. However, a conclusive assessment of the effects of early identification of MCADD on cognitive ability will require considerably longer follow-up. ${ }^{44}$

\section{DISCUSSION}

Newborn screening for MCADD clearly prevents death and disability in many children with the disorder. We conservatively project that one in six children born with MCADD will die in childhood in the absence of screening. Without early asymptomatic detection, at least half of children with MCADD will experience a metabolic crisis, and up to 1 in 10 survivors will develop a serious developmental disability. Conservatively, between one in five and one in four children with MCADD will experience death or severe disability without newborn screening for the disorder. The frequency of milder developmental delay is more difficult to assess but is of concern to parents and poses costs to the affected individuals, families, and society.

These estimates can be used to calculate the number needed to screen to prevent one case of death or serious disability. Taking into account an expected prevalence of 1 in 17,000, 70,000 to 80,000 children would need to be screened for MCADD to prevent one case of death or serious disability. At the national level, screening 4 million U.S. births per year could prevent 50 to 60 premature deaths or cases of disability, assuming strict adherence to disease-management guidelines by both parents and providers, access to comprehensive care and health insurance, and a prompt and efficient system of reporting and follow-up.

\section{ACKNOWLEDGMENTS}

We acknowledge Sabrina Cheng, Harry Hannon, and Charles Venditti for their contributions to this article.

\section{References}

1. Wang SS, Fernhoff PM, Hannon WH, Khoury MJ. Medium chain acyl-CoA dehydrogenase deficiency human genome epidemiology review. Genet Med 1999;1:332339.

2. National Newborn Screening and Genetics Resource Center N. Current Newborn Screening (NBS) Conditions by State. Available at: http://genes-r-us.uthscsa.edu/. Accessed 28 July 2005.

3. Seymour CA, Thomason MJ, Chalmers RA, Addison GM, et al. Newborn screening for inborn errors of metabolism: a systematic review. Health Technol Assess 1997;1: $1-95$.

4. Leonard JV, Dezateux C. Screening for inherited metabolic disease in newborn infants using tandem mass spectrometry. BMJ 2002;324:4-5.

5. Wilcken B, Carpenter K, Wiley V. Neonatal screening for medium-chain acyl-CoA dehydrogenase deficiency. Lancet 2002;359:627-628.

6. American College of Medical Genetics. Newborn screening: towards a uniform panel and system. Contract report to Maternal and Child Health Bureau, Health Resources and Services Administration. Available at: http://mchb.hrsa.gov/screening/. Accessed 25 March 2005
7. Oregon Department of Human Services PHL. Expanded Newborn Screening Disorders. Available at: http://www.ohd.hr.state.or.us/nbs/expand.cfm. Accessed 24 January 2005.

8. Schoen EJ, Baker JC, Colby CJ, To TT. Cost-benefit analysis of universal tandem mass spectrometry for newborn screening. Pediatrics 2002;110:781-786.

9. Gregersen N, Bross P, Andresen BS. Genetic defects in fatty acid beta-oxidation and acyl-CoA dehydrogenases. Molecular pathogenesis and genotype-phenotype relationships. Eur J Biochem 2004;271:470-482.

10. Tanaka K, Gregersen N, Ribes A, Kim J, et al. A survey of the newborn populations in Belgium, Germany, Poland, Czech Republic, Hungary, Bulgaria, Spain, Turkey, and Japan for the G985 variant allele with haplotype analysis at the medium chain Acyl-CoA dehydrogenase gene locus: clinical and evolutionary consideration. Pediatr Res 1997;41:201-209.

11. Maier EM, Liebl B, Roschinger W, Nennstiel-Ratzel U, et al. Population spectrum of ACADM genotypes correlated to biochemical phenotypes in newborn screening for medium-chain acyl-CoA dehydrogenase deficiency. Hum Mutat 2005;25:443-452.

12. Ensenauer R, Winters JL, Parton PA, Kronn DF, et al. Genotypic differences of MCAD deficiency in the Asian population: novel genotype and clinical symptoms preceding newborn screening notification. Genet Med 2005;7:339-343.

13. Andresen BS, Dobrowolski SF, O'Reilly L, Muenzer J, et al. Medium-chain acyl-CoA dehydrogenase (MCAD) mutations identified by MS/MS-based prospective screening of newborns differ from those observed in patients with clinical symptoms: identification and characterization of a new, prevalent mutation that results in mild MCAD deficiency. Am J Hum Genet 2001;68:1408-1418.

14. Gregersen N, Andresen BS, Corydon MJ, Corydon TJ, et al. Mutation analysis in mitochondrial fatty acid oxidation defects: exemplified by acyl-CoA dehydrogenase deficiencies, with special focus on genotype-phenotype relationship. Hum Mutat 2001;18:169-189.

15. Roe CR, Ding J. Mitochondrial fatty acid oxidation disorders. In: Scriver CR, Beaudet AL, Sly WS, Valle D, editors. The metabolic and molecular bases of inherited disease. New York: McGraw-Hill, 2001:2297-2326.

16. Clayton PT, Doig M, Ghafari S, Meaney C, et al. Screening for medium chain acylCoA dehydrogenase deficiency using electrospray ionisation tandem mass spectrometry. Arch Dis Child 1998;79:109-115.

17. Carpenter K, Wiley V, Sim KG, Heath D, et al. Evaluation of newborn screening for medium chain acyl-CoA dehydrogenase deficiency in 275000 babies. Arch Dis Child Fetal Neonatal Ed 2001;85:F105-F109.

18. Zschocke J, Schulze A, Lindner M, Fiesel S, et al. Molecular and functional characterisation of mild MCAD deficiency. Hum Genet 2001;108:404-408.

19. Schulze A, Lindner M, Kohlmuller D, Olgemoller K, et al. Expanded newborn screening for inborn errors of metabolism by electrospray ionization-tandem mass spectrometry: results, outcome, and implications. Pediatrics 2003;111:1399-1406.

20. Yoon HR, Lee KR, Kang S, Lee DH, et al. Screening of newborns and high-risk group of children for inborn metabolic disorders using tandem mass spectrometry in South Korea: a three-year report. Clin Chim Acta 2005;354:167-180.

21. Shigematsu Y, Hirano S, Hata I, Tanaka Y, et al. Newborn mass screening and selective screening using electrospray tandem mass spectrometry in Japan. J Chromatogr B Analyt Technol Biomed Life Sci 2002;776:39-48.

22. Chace DH, Kalas TA, Naylor EW. The application of tandem mass spectrometry to neonatal screening for inherited disorders of intermediary metabolism. Annu Rev Genomics Hum Genet 2002;3:17-45.

23. Pollitt RJ, Leonard JV. Prospective surveillance study of medium chain acyl-CoA dehydrogenase deficiency in the UK. Arch Dis Child 1998;79:116-119.

24. Klose DA, Kolker S, Heinrich B, Prietsch V, et al. Incidence and short-term outcome of children with symptomatic presentation of organic acid and fatty acid oxidation disorders in Germany. Pediatrics 2002;110:1204-1211.

25. Wilcken B, Wiley V, Hammond J, Carpenter K. Screening newborns for inborn errors of metabolism by tandem mass spectrometry. N Engl J Med 2003;348:23042312 .

26. Hoffmann GF, von Kries R, Klose D, Lindner M, et al. Frequencies of inherited organic acidurias and disorders of mitochondrial fatty acid transport and oxidation in Germany. Eur J Pediatr 2004;163: 76-80. Epub 2004 Jan 9.

27. Derks TG, Duran M, Waterham HR, Reijngoud DJ, et al. The difference between observed and expected prevalence of MCAD deficiency in The Netherlands: a genetic epidemiological study. Eur J Hum Genet 2005;13:947-952.

28. Waisbren SE, Albers S, Amato S, Ampola M, et al. Effect of expanded newborn screening for biochemical genetic disorders on child outcomes and parental stress. JAMA 2003;290:2564-2572.

29. Leonard JV, Morris AA. Inborn errors of metabolism around time of birth. Lancet 2000;356:583-587.

30. Wilcken B, Hammond J, Silink M. Morbidity and mortality in medium chain acyl coenzyme A dehydrogenase deficiency. Arch Dis Child 1994;70:410-412.

31. Pollitt RJ, Green A, McCabe CJ, Booth A, et al. Neonatal screening for inborn errors of metabolism: cost, yield and outcome. Health Technol Assess 1997;1:1-202. 


\section{Grosse et al.}

32. Pandor A, Eastham J, Beverley C, Chilcott J, et al. Clinical effectiveness and costeffectiveness of neonatal screening for inborn errors of metabolism using tandem mass spectrometry: a systematic review. Health Technol Assess 2004;8:1-121.

33. Pourfarzam M, Morris A, Appleton M, Craft A, et al. Neonatal screening for mediumchain acyl-CoA dehydrogenase deficiency. Lancet 2001;358:1063-1064.

34. Venditti LN, Venditti CP, Berry GT, Kaplan PB, et al. Newborn screening by tandem mass spectrometry for medium-chain acyl-CoA dehydrogenase deficiency: a costeffectiveness analysis. Pediatrics 2003;112:1005-1015.

35. Chace DH, DiPerna JC, Mitchell BL, Sgroi B, et al. Electrospray tandem mass spectrometry for analysis of acylcarnitines in dried postmortem blood specimens collected at autopsy from infants with unexplained cause of death. Clin Chem 2001;47: 1166-1182.

36. Contribution of selected metabolic diseases to early childhood deaths-Virginia, 1996-2001. MMWR Morb Mortal Wkly Rep 2003;52: 677-679.

37. Wilson CJ, Champion MP, Collins JE, Clayton PT, et al. Outcome of medium chain acyl-CoA dehydrogenase deficiency after diagnosis. Arch Dis Child 1999;80:459-462.

38. Iafolla AK, Thompson RJ, Roe CR. Medium-chain acyl-coenzyme A dehydrogenase deficiency: clinical course in 120 affected children. J Pediatr 1994;124:409-415.

39. Boyle CA, Yeargin-Allsopp M, Doernberg NS, Holmgreen P, et al. Prevalence of selected developmental disabilities in children 3-10 years of age: the Metropolitan Atlanta Developmental Disabilities Surveillance Program, 1991. MMWR CDC Surveill Summ 1996;45:1-14.

40. Ziadeh R, Hoffman EP, Finegold DN, Hoop RC, et al. Medium chain acyl-CoA dehydrogenase deficiency in Pennsylvania: neonatal screening shows high incidence and unexpected mutation frequencies. Pediatr Res 1995;37:675-678.

41. Dezateux C. Newborn screening for medium chain acyl-CoA dehydrogenase deficiency: evaluating the effects on outcome. Eur J Pediatr 2003;162:S25-S28.

42. Nennstiel-Ratzel U, Arenz S, Maier EM, Knerr I, et al. Reduced incidence of severe metabolic crisis or death in children with medium chain acyl-CoA dehydrogenase deficiency homozygous for c. 985A $>$ G identified by neonatal screening. Mol Genet Metab 2005;85:157-159.

43. Zytkovicz TH, Fitzgerald EF, Marsden D, Larson CA, et al. Tandem mass spectrometric analysis for amino, organic, and fatty acid disorders in newborn dried blood spots: a two-year summary from the New England Newborn Screening Program. Clin Chem 2001;47:1945-1955.

44. Holtzman NA. Expanding newborn screening: how good is the evidence? JAMA 2003;290:2606-2608.

45. Sander S, Janzen N, Janetzky B, Scholl S, et al. Neonatal screening for medium chain acyl-CoA deficiency: high incidence in Lower Saxony (northern Germany). Eur J Pediatr 2001;160:318-319.

46. Insinga RP, Laessig RH, Hoffman GL. Newborn screening with tandem mass spectrometry: examining its cost-effectiveness in the Wisconsin Newborn Screening Panel. J Pediatr 2002;141:524-531.

47. Lorey F. California Tandem MS research project. 2004 APHL Newborn Screening and Genetics Testing Symposium. Available at: http://aphl.org/conferences/genetic_testing symposium/MSMSStateandOrganizationreports/MSMS\%20State-Lorey22.pdf. Accessed 17 August 2005.

48. Frazier DM. MS/MS Newborn Screening in NC 1997-2003: Outcomes and Predictive Values of Elevated Results, APHL. Available at: http://aphl.org/conferences/genetic testing_symposium/MSMSStateandOrganizationreports/MSMS\%20State-Frazier25.pdf. Accessed 17 August 2005.

49. Annual Report, Wadsworth Center, New York State Department of Health. Available at: http://www.wadsworth.org/newborn/annualrept/annsum.htm. Accessed $24 \mathrm{Au}-$ gust 2005 .

50. Annual Report, Indiana State Department of Health Available at: http://www. state.in.us/isdh/programs/nbs/NBSStatistics.htm. Accessed 24 August 2005.

51. Touma EH, Charpentier C. Medium chain acyl-CoA dehydrogenase deficiency. Arch Dis Child 1992;67:142-145. 